\title{
Supporting Information : Paper-based Optode Devices (PODs) for Selective Quantification of Potassium in Biological Fluids
}

\author{
Daniel B. Lookadoo, ${ }^{*+}$ Jeremy E. Schonhorn, ${ }^{+}$Harshit Harpaldas, ${ }^{\dagger}$ Christopher M. Uherek, ${ }^{\dagger}$ Philipp \\ Schatz, $₫$ Anna Lindgren, $\$$ Michal Depa, ${ }^{\dagger}$ Ashok A. Kumar ${ }^{\dagger}$ \\ †Jana Care, Inc., Boston, Massachusetts 02215, United States \\ ‡Precision Medicine \& Biosamples, R\&D, AstraZeneca, Gothenburg, Sweden \\ *Corresponding author: dlookadoo@janacare.com
}

\section{METHODS AND MATERIALS}

\section{Chemicals}

Valinomycin, sodium tetrakis[3,5-bis-(trifluoromethyl) phenyl]borate (BARF), 3-octadecanoylimino-7-diethylamino-1,2benzophenoxazine ( $\mathrm{CH} \mathrm{I}$ ), Pluronic F-127 (F-127), 9-(Diethylamino)-5-[(2-octyldecyl)imino]benzo[a]phenoxazine (CH III), bis(2ethylhexyl) decanedioate (DOS), and sucrose were obtained from Sigma-Aldrich. 2-[bis(2-hydroxyethyl)amino]-2(hydroxymethyl)propane-1,3-diol (Bis-tris), N,N-Bis(2-hydroxyethyl)-2-aminoethanesulfonic acid (BES), tetrahydrofuran (THF), potassium chloride $(\mathrm{KCl})$, lithium chloride $(\mathrm{LiCl})$, and sodium chloride $(\mathrm{NaCl})$ were obtained from Acros Organics. Water (Ultra trace grade), nitric acid (ICP-OES grade), and were obtained from Thermo Scientific. 1000 ppm potassium was obtained from Inorganic Ventures. Ox Blue (Ox B) was synthesized as previously described. ${ }^{1}$

\section{Optode Synthesis}

We explored similar molar ratios and addition volumes as those described in the literature ${ }^{2-4}$ An optode cocktail was prepared by dissolving an ionophore, ion-exchanger, chromoionophore, DOS, and F-127 in THF. The presented method used a cocktail prepared by dissolving Valinomycin (7.5 mg), BARF (4.1 mg), CH1 (2.2 mg), F-127 (0.5 mg), and DOS (5.4 mg) in 0.5 mL of THF. Optodes were prepared by adding $0.5 \mathrm{~mL}$ of cocktail to $1.5 \mathrm{~mL}$ of water while vortexing for 30 seconds. Optodes used for in solution titration experiments were blown with compressed air on the surface for 30-45 minutes to remove THF and concentrate the solution. Optodes used to prepare paper optodes were used without THF removal and $0.5 \mathrm{~mL}$ of a $50 \%$ (wt/vol) sucrose solution was added prior to dispensing. Optodes were concentrated by diluting $2.0 \mathrm{~mL}$ of optode solution with $3.0 \mathrm{~mL}$ of a $0.1 \%$ (wt/vol) F-127 solution and reduced to the desired volume using Amicon Ultra centrifugal filters (Millipore).

\section{Fabrication of the Paper-based Devices}

PODs were cut from cards fabricated by stacking a spreading mesh, sample pad, and optode paper. Spreading mesh (Hyphyl 285/44, Saati) was trimmed into $8 \mathrm{~mm}$ by $300 \mathrm{~mm}$ strips. Sample pads were prepared by dispensing $1.0 \mathrm{~mL}$ of $50 \mathrm{mM}$ Bis-Tris/BES pH 7.4 buffer with $10 \%(\mathrm{w} / \mathrm{v}$ ) sucrose onto a $8 \mathrm{~mm}$ by $300 \mathrm{~mm}$ strip of CytoSep HV (Ahlstrom) and drying for 20 minutes in a forced air oven at $70^{\circ} \mathrm{C}$. Optode paper was prepared by dispensing the optode solution onto a $8 \mathrm{~mm}$ by $300 \mathrm{~mm}$ strip of paper (Whatman CF1) at a dispense rate of $1.5 \mu \mathrm{L} / \mathrm{mm}$ using an IsoFlow reagent dispensing system (Imagene Technology), and drying for 6 minutes in a forced air oven at $70^{\circ} \mathrm{C}$. The stack of materials was sandwiched in-between recycled polyethylene foam spacers (FLEXcon) and adhered to a 10 mil PET backing (Lohmann Tapes) to provide structure with packing tape (Duct HP260).

CorelDraw was used to design a repeating pattern, of $4 \mathrm{~mm}$ holes interspaced by $6.5 \mathrm{~mm}$, and a Helix Laser Machine (Epilog Laser) was used to cut the pattern into the PET backing to generate a sample port. Individual layers were stacked and laminated using a Universal Laminator Module (Kinematic Automation). Assembled cards were cut into individual PODs using a Matrix 2360 Programmable Shear (Kinematic Automation). 


\section{Sample Preparation and Measurements}

$\mathrm{K}^{+}$depleted plasma was prepared via size-exclusion chromatography. Lithium heparin plasma (Research Blood Components), pooled from 10 donors, was buffer exchanged into $12 \mathrm{mM}$ phosphate buffer pH 7.4 with $150 \mathrm{mM} \mathrm{Na}^{+}$and $129 \mathrm{mM} \mathrm{Cl}^{-}$using a PD10 column (GE). The residual $\mathrm{K}^{+}$concentration was determined by Atomic Emission Spectroscopy (AES). Elevated $\mathrm{K}^{+}$samples were obtained by spiking the depleted plasma with $\mathrm{KCl}$ to achieve the desired concentration. $\mathrm{K}^{+}$measurements with paper optodes and PODs were performed using 2.5 and $20 \mu \mathrm{L}$ of sample, respectively. The optical value was recorded after $\sim 30$ seconds and the difference from the measurement prior to sample application was calculated.

Fresh lithium heparin whole blood (Research Blood Components) was used to obtain a native and elevated $\mathrm{K}^{+}$sample. The elevated whole blood sample was obtained by spiking the native sample with $\mathrm{KCl}$ to achieve the desired concentration. The native and spiked sample concentrations were determined by ISE. $\mathrm{K}^{+}$measurements made with whole blood and PODs were performed using $20 \mu \mathrm{L}$ of sample. The optical value was recorded after 90 seconds and the difference from the measurement prior to sample application was calculated.

\section{Instrumentation and Measurements}

Absorbance measurements of optode solutions were made using a DH-2000-BAL light source with a SQUARE ONE cuvette holder coupled to a Flame UV-Vis spectrometer (Ocean Optics). Reflectance measurements of paper optodes were made using a ISP-REF integrating sphere coupled to a Flame UV-Vis spectrometer (Ocean Optics). Reflectance measurements of PODs were made using a handheld reader (Jana Care). Atomic emission measurements were made using a 4200 MP-AES (Agilent Technologies). Ionselective Electrode measurements were made using a Medica EasyLyte Analyzer.

Table S1. Statistical Summary of the Feasibility Assessment including: Expected and Measured Mean $\mathrm{K}^{+}$Concentration in $\mathrm{mM}$, and Relative Error (Rel. Error) and Relative Standard Deviation (rsd) as a Percentage.

\begin{tabular}{|c|c|c|c|}
\hline Expected & Mean & Rel. Error & rsd \\
\hline 2.10 & 1.9 & -7.4 & 8.1 \\
\hline 3.10 & 3.3 & +7.4 & 6.0 \\
\hline 4.10 & 4.3 & +5.2 & 4.1 \\
\hline 5.10 & 4.8 & -6.2 & 4.5 \\
\hline 6.10 & 6.4 & +5.1 & 3.5 \\
\hline 7.10 & 6.9 & -2.8 & \\
\hline
\end{tabular}

\section{EXPERIMENTAL DETAILS}

\section{Characterization of Filter Paper}

The presence of red blood cells (RBCs) can produce issues with sensor biofouling and introduce optical interference. An estimate of $0.11 \pm 0.01 \%$ was obtained for the degree of on-device hemolysis by evaluating the quality of the filtered plasma from 30 devices, according to Scheme 1, and determining the degree of hemolysis introduced via filtration. The impact on optical signal-to-noise was assessed, as illustrated in Figure S1, and found to be insignificant across the measuring range. 
Scheme S1. Schematic illustration of the on-device hemolysis analysis. The blood sample is applied to the device, allowed to develop, and then disassembled to remove the optode paper-containing the filtered plasma. The plasma is extracted from the optode paper in buffer and then concentrated using centrifugal filters for hemoglobin measurement. ${ }^{5}$ The level of on-device hemolysis is estimated by determining the amount of hemoglobin in the plasma and comparing it to the total hemoglobin in the sample.

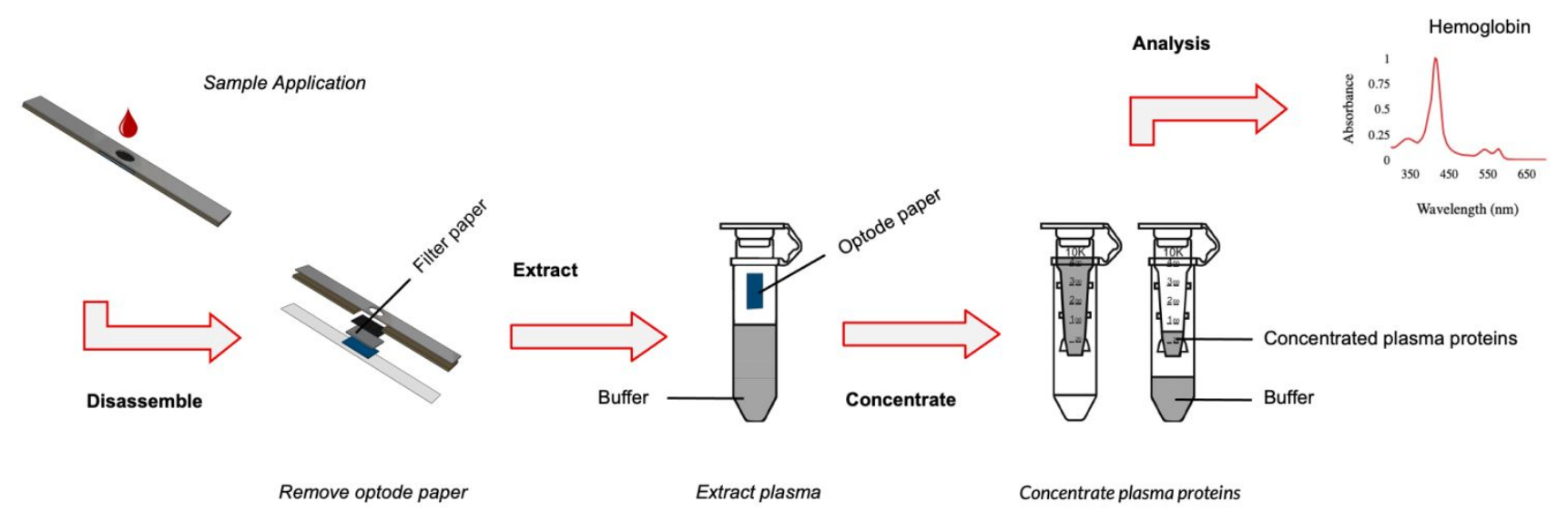

a

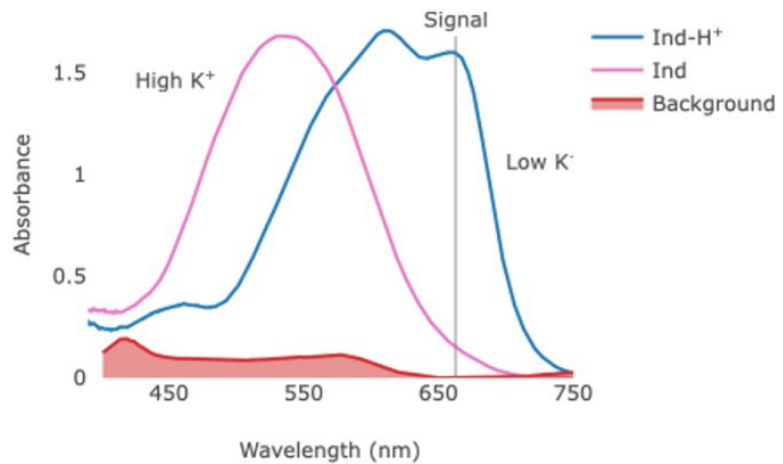

b

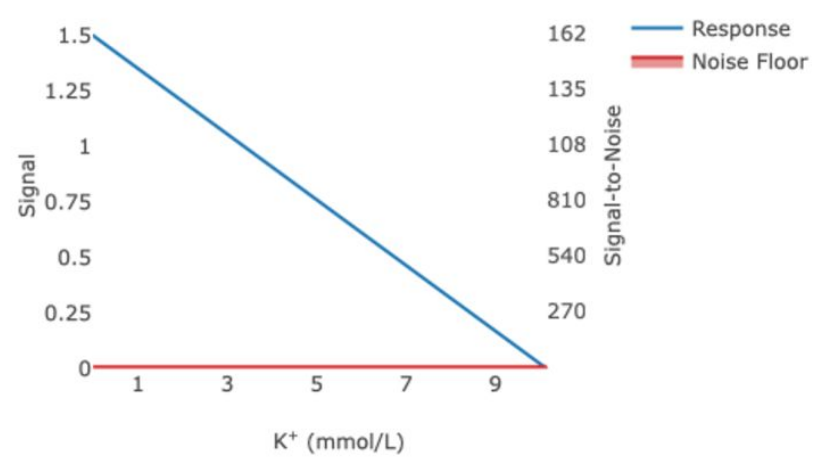

Figure S1. Plots illustrating the assessment of optical signal-to-noise. (a) Absorbance spectra for both forms of the chromoionophore (CH I) and the background. (b) Signal-to-noise plot for the $\mathrm{K}^{+}$response (Signal), based on the calibration curve, and the background due to whole blood components (Noise). The noise floor is equivalent to a signal-to-noise equal to 10 . 


\section{REFERENCES}

1. Xie, X.; Crespo, G. A.; Bakker, E., Oxazinoindolines as fluorescent $\mathrm{H}+$ turn-on chromoionophores for optical and electrochemical ion sensors. Anal. Chem. 2013, 85 (15), 7434-7440.

2. Gerold, C. T.; Bakker, E.; Henry, C. S., Selective Distance-Based K(+) Quantification on Paper-Based Microfluidics. Anal Chem 2018, 90 (7), 4894-4900.

3. Xie, X.; Crespo, G. A.; Zhai, J.; Szilagyi, I.; Bakker, E., Potassium-selective optical microsensors based on surface modified polystyrene microspheres. Chem Commun (Camb) 2014, 50 (35), 4592-5.

4. Xie, X.; Mistlberger, G.; Bakker, E., Ultrasmall fluorescent ion-exchanging nanospheres containing selective ionophores. Anal. Chem. 2013, 85 (20), 9932-9938.

5. Harboe, M., A method for determination of hemoglobin in plasma by near-ultraviolet spectrophotometry. Scand J Clin Lab Invest 1959, $11,66-70$ 